\title{
Principios de Desarrollo Profesional Docente construidos por y para Profesores de Ciencia: una propuesta sustentable que emerge desde la indagación de las propias prácticas*
}

\author{
Principles of Teacher Professional Development built by and for Science Teachers: a \\ sustainable proposal that emerges from the inquiry of self-practices
}

Princípios de desenvolvimento profissional docente construídos por e para professores de Ciências: uma proposta sustentável que emerge a partir da indagação das próprias práticas

\section{Corina González-Weil, ${ }^{a}$ Melanie Gómez Waring, ${ }^{b}$ Germán Ahumada Albayay, ${ }^{c}$ Paulina Bravo González, ${ }^{d}$ Exequiel Salinas Tapia, ${ }^{e}$ Damián Avilés Cisternas, ${ }^{f}$ José Luis Pérez, ${ }^{g}$ Jonathan Santana Valenzuela ${ }^{h}$}

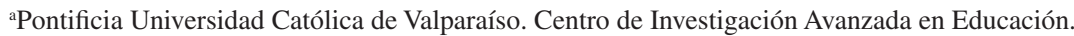 \\ Telf.: (562)322274859. Correo electrónico: corina.gonzalez@ucv.cl \\ bPontificia Universidad Católica de Valparaíso. Escuela Santa Julia, Viña del Mar \\ 'Pontificia Universidad Católica de Valparaíso. Liceo José Cortés Brown, Viña del Mar \\ dPontificia Universidad Católica de Valparaíso. Instituto Técnico Profesional Marítimo de Valparaíso \\ éciceo Politécnico de Concón. Colegio Cardenal Silva Henríquez, Viña del Mar \\ ${ }^{\mathrm{f} C}$ Colegio Los Leones, Quilpué \\ gPontificia Universidad Católica de Valparaíso. Liceo María Luisa Bombal, Valparaíso \\ hPontificia Universidad Católica de Valparaíso
}

\begin{abstract}
RESUMEN
La transformación de las prácticas de los docentes en el área de ciencias, tanto a nivel escolar como universitario (incluida la formación inicial), constituye una necesidad urgente si pretendemos alfabetizar científicamente a la población. Este estudio muestra una propuesta de cinco principios para el desarrollo profesional docente en ciencias, que emergen desde la indagación colaborativa de las propias prácticas de un conjunto de docentes de educación primaria, secundaria y universitaria, quienes trabajan hace cuatro años en un proceso de desarrollo profesional conjunto. Estos principios incluyen la construcción de una visión común acerca del para qué enseñar ciencias, la indagación de las prácticas a partir de las particularidades de la educación científica, la reflexión individual y colectiva sobre las prácticas, la valoración de la autoridad de la experiencia para el aprendizaje docente y la promoción de un ambiente de desarrollo profesional que involucre diversidad de contextos y niveles de enseñanza.
\end{abstract}

Palabras clave: educación científica, desarrollo profesional docente, indagación como postura.

\begin{abstract}
The transformation of teaching practices in the area of sciences, both at school and university levels (including initial training), is an urgent need if we intend to achieve scientific literacy in the population. This study shows a proposal of five principles for teacher professional development in sciences, emerged from the collaborative inquiry of self-practices in a group of primary, secondary and university teachers, who have been working together for four years in a professional development process. These principles include the construction of a common
\end{abstract}

Este trabajo ha sido financiado por el Centro de Investigación Avanzada en Educación (CONICYT-CIE 05). Agradecemos a esta instancia, así como a los establecimientos, docentes, colegas y estudiantes que participaron de este estudio por el apoyo prestado. 
vision on the purpose for teaching sciences; the inquiry of practices starting from the distinctive features of scientific education; individual and collective reflection on practices; the valuation of the authority of experience for teacher learning; and the promotion of an environment of professional development involving diverse contexts and teaching levels. Implications for professional development are discussed.

Key words: science education, professional development, inquiry as stance.

\section{RESUMO}

A transformação das práticas dos docentes na área das ciências, tanto a escolar como a universitária (incluindo formação inicial), constitui uma necessidade urgente se pretendemos alfabetizar cientificamente a população. Mostra uma proposta de 5 princípios para o desenvolvimento profissional docente em ciências, a partir da indagação colaborativa das práticas de um grupo de docentes de educação primária, secundária e universitária que trabalha, conjuntamente, em um processo de desenvolvimento profissional, há quatro anos. Esses princípios incluem a construção de uma visão comum sobre o para quê ensinar ciências, sobre a indagação das práticas a partir das particularidades da educação científica, sobre a reflexão individual e coletiva a cerca de tais práticas, sobre a valoração da autoridade da experiência para a aprendizagem docente e sobre a promoção de um ambiente de desenvolvimento profissional que envolva uma diversidade de contextos e níveis de ensino.

Palavras-chave: educação científica, desenvolvimento profissional docente, indagação como postura.

\section{INTRODUCCIÓN}

En general, la formación inicial de profesores parece tener una escasa influencia en cómo los docentes enseñamos en el aula (Latorre, 2005). Si bien durante la formación inicial se abordan muchos contenidos, a menudo la manera en que enseñamos -centrada en el profesor- se contradice con cómo queremos que enseñen los futuros profesores -centrada en el alumno- (Munby y Russell, 2003). Esto podría estar dado por las creencias que se manifiestan al interior de los programas de formación, como pensar que para una enseñanza efectiva basta el conocimiento del contenido y que éste se traspasa al estudiante (Clough, Berg y Olson, 2009). Como consecuencia, la formación inicial a menudo promueve la replicación de un tipo de enseñanza descontextualizada y con énfasis en el contenido (Echeverría, 2010; Cofré et al., 2010). Así, no es de extrañar que al momento de egresar, los docentes recurramos a los recuerdos que tenemos de nuestros propios profesores durante la etapa escolar y universitaria, o bien, reproduzcamos nuestras propias prácticas y la de nuestros colegas (Latorre, 2005). Desde esta perspectiva, los procesos de desarrollo profesional continuos, se vuelven críticos y desafiantes si se pretende realmente lograr un proceso efectivo de mejoramiento de la enseñanza. En el caso de la Educación en Ciencias, estos procesos se vuelven especialmente relevantes, si consideramos que existe consenso en que el objetivo actual de ésta se sitúa desde el concepto de Alfabetización Científica, es decir el desarrollo de conocimientos, capacidades y actitudes que permitan a los individuos enfrentar, negociar y tomar decisiones en situaciones cotidianas relacionadas con la Ciencia (Sadler, 2011). No obstante, los resultados de nuestros estudiantes en pruebas como PISA y TIMSS y las altas tasas de reprobación de asignaturas científicas a nivel universitario, muestran que el objetivo de alfabetizar científicamente a nuestros estudiantes está lejos de ser alcanzado. Una de las razones de ello es la mantención de una enseñanza frontal, descontextualizada y contenidista de las Ciencias. En palabras de Albertini et al., la enseñanza de las ciencias "ha girado tradicionalmente en torno de una enseñanza desagregada o disciplinaria del saber científico, una instrucción enciclopedista, un aprendizaje memorístico de conocimientos atomizados, datos fragmentarios e informaciones puntuales, con una comprensión de la 
ciencia descontextualizada del mundo cotidiano y de las necesidades de la vida social" (Albertini et al., 2005: 46).

A partir de lo anterior, surge la urgente necesidad de reformar la educación en ciencias, y en particular, transformar las prácticas docentes, tanto en la Escuela como en la Universidad. Pero ¿bajo qué condiciones un docente transforma sus prácticas? Si bien ha habido diversas iniciativas de desarrollo profesional docente orientadas a reformar las prácticas, éstas se han llevado a cabo, demasiado a menudo, desde una perspectiva vertical, distinguiendo a un Ministerio/Universidad de carácter técnico que diseña y una Escuela o Liceo que recibe la intervención, sin mayor incidencia en el proceso (Muñoz y Vanni, 2008) y sin dar mayores espacios para la reflexión docente y el trabajo colaborativo entre pares.

El presente trabajo se origina en un proyecto que, en un inicio, pretendía desarrollar, en conjunto con un grupo de profesores secundarios de ciencia, una propuesta de desarrollo profesional. Esta línea de trabajo nació de la convicción de que la educación en ciencias ayuda a la movilidad social, pero también a convertir a los estudiantes en agentes activos en la transformación de sus propias comunidades (ver González-Weil et al., 2009). Continuó con el estudio de lo que un conjunto de destacados profesores de ciencia secundarios chilenos hacen en el aula (ver González-Weil et al., 2012), lo que permitió visualizar elementos que podrían ser relevantes para la formación continua. Luego, describimos cómo es que ese proceso de formación continua podría tener lugar, a través del relato de una experiencia concreta de desarrollo profesional (ver González-Weil et al., 2013). Lo que aquí presentamos es la mirada a un cuarto momento de este proceso. Producto del trabajo colegiado de una diversidad de docentes de ciencia es que surge una propuesta de 5 Principios para el Desarrollo Profesional Docente, pensados a partir de un proceso de indagación de nuestras propias prácticas. Al finalizar, presentaremos algunas reflexiones acerca de futuras propuestas de desarrollo profesional, así como de la manera en que pensamos debiera darse la relación entre la Universidad y la Escuela.

\subsection{DESARROLLO PROFESIONAL EFECTIVO: UNA TAREA PENDIENTE}

Núñez, Arévalo y Ávalos (2012: 12) señalan como uno de los grandes problemas del desarrollo profesional docente (DPD) el "desencuentro entre ámbitos de formación (inicial y continua), ámbitos del saber (teoría y práctica, saberes fundamentales y saberes de experiencia) y unidades formadoras (universidad y escuela)” y se pregunta si acaso será posible concebir y ejecutar una propuesta que congregue estos distintos ámbitos. Históricamente, pareciera ser que estos ámbitos han estado escindidos y han corrido por carriles paralelos. Tradicionalmente y a nivel global, los modelos de DPD han sido estructurados en su mayoría desde la Universidad o desde las instancias ministeriales, y elaborados desde una perspectiva más bien teórica, más que generarse de manera conjunta con la Escuela o considerando la perspectiva práctica, el saber de los docentes y sus necesidades. Así, muchos procesos de formación continua responden a modelos de "racionalidad técnica", donde el profesor es un "técnico" que debe aplicar los métodos generados por la investigación. Esto supone que la función del docente es "verificar" lo que el investigador produce, de manera análoga a las visiones positivistas de la ciencia, que establecen que el conocimiento es acumulativo y el nuevo conocimiento debe ser probado a través del método científico empirista (Mellado, 2001). Desde esta perspectiva, existe un experto que tiene una "verdad", la cual transmite al docente para que la aplique 
en el aula. Estos modelos desconocen las implicaciones éticas y políticas del quehacer educativo (De Lella, 1999), y han mostrado ser altamente ineficaces para cambiar, tanto las concepciones como las prácticas de los docentes (Mellado, 2001). Bajo este contexto, Ávalos (2002) señala que la dificultad que tienen los docentes para innovar sus prácticas y asumir reformas, demuestra que quienes las diseñan no consideran que el cambio docente es en sí mismo un proceso de aprendizaje, y que como tal, debe estar enraizado en la práctica cotidiana del profesor.

No obstante lo anterior, pareciera haber -a nivel internacional- una tendencia a alejarse de estos procesos de desarrollo profesional tradicionales/verticales, promoviendo instancias que posibilitan una mayor interacción entre docentes y una inclusión de las experiencias de práctica como objeto fundamental de reflexión para la mejora. Bajo este contexto, hay ciertos hallazgos que respaldan las características que debieran tener modelos más alternativos de formación: estos incluyen la generación de oportunidades para el coaprendizaje entre docentes, procesos de formación prolongados y de largo plazo (más que instancias puntuales), y la inclusión de aprendizaje y de reflexión sobre las propias prácticas, donde esta última aparece como un instrumento promotor del cambio (Ávalos, 2011). Un estudio de Schnellert et al. (2008, cit. en Ávalos, 2011: 17), señala que los cambios en las prácticas docentes, más que estar relacionados a los años de experiencia, estarían gatillados por el grado de involucramiento de los docentes en una indagación reflexiva y colaborativa. Así, la reflexión docente pareciera ser el factor clave en la transformación de las prácticas: un docente que no problematiza su práctica y no reflexiona sobre ella, no sentirá la necesidad de actuar de manera diferente en el aula.

Por otra parte, la retroalimentación, el acceso a nuevas ideas e información se posibilita a través de la interacción y el diálogo con otras personas en el marco del trabajo colaborativo, el cual, a su vez, contribuye a crear una cultura de aprendizaje y una comunidad, en donde el aprendizaje es valorado y estimulado (Chou, 2011). El trabajo docente en torno a estas comunidades de aprendizaje incidirían, además, positivamente en el aprendizaje de los estudiantes de los docentes participantes (Vescio, Ross y Adams, 2008). Finalmente, procesos de investigación o indagación de la propia práctica, con el propósito de comprender y mejorar el propio quehacer profesional, se prestan como un escenario adecuado para potenciar la reflexión a través de la colaboración. Estos procesos de investigación - por ejemplo, a través de una Investigación-Acción- son considerados una forma efectiva de desarrollo profesional docente, especialmente cuando se realiza de manera colaborativa (Lebak y Tinsley, 2010; Ponte, 2010).

Para finalizar, quizás la característica más distintiva de los procesos de DPD que se promueven hoy en día se relaciona con el generar en el docente una postura de reflexión y transformación de su práctica. Cochran-Smith y Lytle se refieren con ello a la "adquisición de una actitud o postura indagatoria frente a la enseñanza" (2003: 70), donde los profesores se cuestionan sus propios conocimientos y su práctica, y colaboran en el cuestionamiento de los conocimientos y las prácticas de otros para transformar su práctica desde una postura crítica. Esta idea se condice ampliamente con aquella que señala que el docente debe tener una actitud de aprendizaje permanente, no sólo en relación a actualizarse en la disciplina que enseña, sino que también a aprender de su propia práctica. En el caso de los docentes de ciencia, se utiliza el concepto de profesor de ciencias como aprendiz (Loughran, 2007). Esta actitud de permanente aprendizaje debiera también ser modelada en los procesos de formación inicial, por lo que es igualmente aplicable a los formadores de profesores. Tanto 
en el caso de los profesores de ciencia en formación o en ejercicio, como en el caso de los formadores de profesores, la actitud de profesor de ciencias como aprendiz involucra la acción de recoger y analizar evidencia acerca de las propias prácticas y el efecto que éstas tienen sobre sus alumnos.

\subsection{DESARROLLO Y APRENDIZAJE PROFESIONAL DE DOCENTES DE CIENCIAS}

En 1996, Beverley Bell y John Gilbert proponen un modelo para el desarrollo profesional docente basado en un estudio de 3 años, realizado en Nueva Zelanda. El estudio documenta cómo un conjunto de docentes de ciencia cambia sus prácticas desde un enfoque transmisivo hacia un enfoque que ayuda a los estudiantes a construir su propio conocimiento, a través del cuestionamiento de sus ideas previas y la reflexión sobre su aprendizaje (Simon y Campbell, 2012). El modelo propone tres ámbitos de desarrollo o aprendizaje profesional, que se relacionan entre sí: el ámbito personal, que involucra sentimientos, motivación, disponibilidad y compromiso del profesor; el ámbito profesional que referiere conocimientos y concepciones, así como visualización de los efectos de la práctica en los estudiantes; y ámbito el social, correspondiente a la valoración del trabajo colaborativo y el contexto de trabajo (Mellado, 2001). Este modelo también propone una cierta progresión: primero, el docente ve algún aspecto de su práctica como problemático (ámbito personal), luego visualiza que es un problema el trabajar de manera aislada (ámbito social) y, finalmente, se motiva a probar nuevas maneras de enseñar (ámbito profesional). A medida que progresa, el profesor debe lidiar con los sentimientos y preocupaciones que involucran el cambio de sus prácticas, y comienza a cambiar su idea de lo que significa ser un profesor de ciencias (ámbito personal). Del mismo modo comienza a valorar el trabajo colaborativo (social) y tiene confianza para implementar sus propias ideas en su clase (profesional). Finalmente, el docente se siente empoderado lo que aumenta su autoconfianza (personal), comienza a buscar activamente colaboración (social) y eventualmente desarrolla nuevas actividades de desarrollo profesional (profesional) (Simon y Campbell, 2012).

El modelo que Bell y Gilbert proponen es poderoso y relevante porque ilustra con evidencias el hecho de que el cambio de las propias prácticas, necesariamente va asociado a un proceso de reconstrucción profunda del significado de lo que es ser un profesor de ciencias (Simon y Campbell, 2012). Según Bell (1998), esta reconstrucción ocurre en interacción con otros docentes, y es esta re-significación del sentido de enseñar ciencias lo primero que debe suceder para que ocurra la innovación de las prácticas. En el contexto chileno, un ejemplo de lo anterior podría ser que en vez de que un profesor tenga como objetivo principal el elevar el resultado en las pruebas estandarizadas de sus estudiantes, transite hacia una mirada que también incluya como objetivo el desarrollo de conocimientos y habilidades científicas, que permitan a sus alumnos tomar decisiones informadas en asuntos relacionados con la Ciencia.

En cuanto a los elementos que favorecen el desarrollo o aprendizaje profesional de los docentes de ciencias, Hoban (2002, cit. en Simon y Campbell, 2012) identifica 8 condiciones: (a) una concepción de enseñanza como una relación dinámica con los estudiantes y con otros colegas, que implica incerteza y ambigüedad al momento de cambiar; (b) espacio para la reflexión, para poder comprender los cambios que se generan; (c) un objetivo claro que promueva el deseo de cambiar; (d) una comunidad para intercambiar experiencias; (e) oportunidades de probar qué funciona y qué no en el aula; (f) referentes conceptuales 
que contribuyan a extender el conocimiento y la experiencia; (g) retroalimentación de los estudiantes en respuesta a las nuevas prácticas implementadas; (h) suficiente tiempo para adaptarse a los cambios que se realizan. Lo anterior, coincide en parte con lo que los investigadores norteamericanos Loucks-Horsley, Stiles y Hewson publicaran en 1996, quienes, basados en una acuciosa revisión, proponen Siete Principios para un Desarrollo Profesional Efectivo (DPE) para profesores de Matemáticas y Ciencia, los que, a la fecha, aún son un referente (Chval, et al., 2008). De manera muy resumida, esta propuesta de DPE plantea las siguientes condiciones: (1) debe estar guiado por una nítida y clara imagen de enseñanza efectiva que incluye, entre otros, un énfasis en el aprendizaje a través de la resolución de problemas y la investigación del estudiante, el desarrollo de la comprensión en profundidad de conceptos clave y el trabajo colaborativo; (2) entrega a los profesores oportunidades para desarrollar conocimiento y habilidades para crear mejores condiciones para el aprendizaje a sus estudiantes; (3) los métodos utilizados para enseñar a los docentes han de ser un reflejo de los métodos que los profesores han de usar con sus propios estudiantes; (4) se construye sobre la base de comunidades de aprendizaje, a través del intercambio de opiniones y el trabajo colaborativo; (5) prepara a los docentes para ser líderes, proveyéndolos de oportunidades de planificar e implementar oportunidades de desarrollo profesional para sí mismos y para sus colegas, actuando como agentes de cambio y apoyando los procesos de desarrollo de otros profesores; (6) provee a los docentes de vínculos a otros estamentos del sistema educativo, estableciendo redes al interior y al exterior del establecimiento y alineándose con estándares educativos nacionales; (7) incluye una evaluación continua que determine en todo momento el grado de satisfacción de los docentes, evalúe a largo plazo su impacto y monitoree la experiencia de desarrollo profesional, de modo de ir mejorándola de manera continua (Chval et al., 2008; LoucksHorsley, Stiles y Hewson 1996).

En resumen, los procesos de desarrollo profesional en profesores de ciencia, deben involucrar la generación de espacios y contextos en donde los docentes puedan resignificar el sentido que le dan a la enseñanza de las ciencias; cuestionar sus propias creencias acerca de la ciencia, su enseñanza, y su aprendizaje; probar nuevas metodologías y, por sobre todo, reflexionar acerca de sus prácticas de manera colaborativa.

\section{METODOLOGÍA}

\subsection{CONTEXTO}

Los principios presentados en el presente trabajo surgen inicialmente como orientaciones de una propuesta de formación continua que un grupo de profesores de ciencia de nivel secundario, junto al equipo de investigación del Laboratorio de Didáctica de la Biología de la Pontificia Universidad Católica de Valparaíso, elaboró durante el año 2012. Este grupo de docentes ya había participado en un proceso anterior de formación que tenía como foco una investigación-acción de las propias prácticas (ver González-Weil et al., 2013). A partir de esa primera experiencia de formación, la mayoría de los profesores participantes decidió continuar reuniéndose junto al equipo, resultando -además de las orientaciones señaladas- una postulación a un proyecto en donde académicos y profesores fueron co-investigadores. 
El presente artículo es fruto de dicho proyecto y se genera en el seno de una segunda experiencia de formación continua, de la cual participa la mayor parte del grupo original de docentes, además de colegas nuevos que fueron invitados al grupo. Este conjunto de docentes, del que los autores formaron parte activa, se reunieron cada 15 días entre marzo de 2013 a abril 2014 (16 sesiones en total, de 3 horas cada una), en dependencias de la Universidad. El objetivo del proceso era indagar colaborativamente la manera de mejorar nuestras prácticas, tanto escolares como universitarias, a través de la comprensión de un problema común que aquejara nuestras prácticas docentes en la enseñanza de las ciencias (o en el caso de los formadores de profesores, a la enseñanza de la educación en ciencias), para luego, en conjunto, explorar y tomar acciones de mejora. La pregunta inicial del grupo fue: ¿cómo respondemos a las necesidades de los alumnos y de la comunidad local a través de la educación en ciencias? No obstante, la discusión acerca de las necesidades de nuestros estudiantes y la búsqueda de aspectos que pudiéramos abordar en común en los diferentes contextos, nos llevó a pensar en una meta que conjugara la promoción de aprendizajes conceptuales con las habilidades científicas. De esta forma, surgió la creatividad como una de las características básicas del quehacer científico y a su vez, uno de los aspectos menos utilizados en las aulas de ciencia, el grupo decidió proponerse como meta común el cómo llevar la creatividad a la sala de clases. Lo anterior generó el compartir experiencias y discutir sobre cómo se presenta y puede implementarse el tema de la creatividad en procesos de enseñanza y aprendizaje de las ciencias, entendiendo que a través de su promoción y uso se ampliaban las posibilidades de desarrollar aprendizajes en los estudiantes. Paralelamente, se trabajó la temática de cómo vincular la educación en ciencias con el contexto y, de ser posible, con las necesidades de la comunidad local a través de la exploración del uso de cuestiones socio-científicas (ver Sadler, 2011). Finalmente, contamos con la visita de algunos investigadores extranjeros ${ }^{1}$, quienes intercambiaron con el grupo experiencias y conocimiento respecto a la generación de conocimiento en comunidades de aprendizaje, concepciones alternativas, argumentación científica, formación inicial, entre otros. Es en este nuevo contexto de formación profesional basado en la promoción de una postura indagatoria de las prácticas (Cochran-Smith y Lytle, 2003), en el cual emergen los principios para el desarrollo profesional docente para profesores de ciencia que aquí se proponen.

\subsection{PARTICIPANTES}

El presente artículo reporta el trabajo realizado por un grupo de aproximadamente 23 docentes de ciencia y educación en ciencias, quienes se distribuyen en: 14 docentes de ciencia secundarios ( 3 de química, 6 de física, 3 de biología, 1 de mecánica automotriz, 1 de matemáticas); 2 docentes de ciencia de educación básica; 4 docentes universitarios de ciencia y/o formación de profesores de ciencia ${ }^{2}, 4$ docentes de ciencia en formación ${ }^{3}$ y una bióloga como parte del equipo de investigación.

Nos visitaron: Ian Mitchell, Universidad de Monash, Australia y co-fundador del proyecto PEEL (Project for Enhancing of Effective Learning); Tom Russell, Universidad de Queens, Canadá; María Pilar Jimenez-Aleixandre Universidad de Santiago de Compostela, España y Elio Ricardo, Universidad de Sao Paulo, Brasil.

2 De estos cuatro docentes universitarios, uno es psicólogo y dos trabajan además en la Escuela: uno es profesor de física y otra es profesora de educación básica.

Estudiantes de último año de Pedagogía en Biología y Ciencias Naturales y de Pedagogía en Física. 
Estudios Pedagógicos, vol. XL, Número Especial 1: 105-126, 2014

PRINCIPIOS DE DESARROLLO PROFESIONAL DOCENTE CONSTRUIDOS POR Y PARA PROFESORES DE CIENCIA: UNA PROPUESTA SUSTENTABLE QUE EMERGE DESDE LA INDAGACIÓN DE LAS PROPIAS PRÁCTICAS

\title{
2.3. COLECCIÓN Y ANÁLISIS DE DATOS
}

De manera intercalada a las sesiones regulares del grupo, un subconjunto de este equipo correspondiente a co-investigadores y tesistas, nos reunimos para evaluar el avance de las sesiones y sistematizar la información generada por el grupo. Los datos se recogieron a partir de diferentes fuentes: (a) filmación de todas las sesiones, registro que sirvió de referencia para el análisis general; (b) documentos generados por los docentes participantes, como escritura de historias de clase (ver "narrativa como texto experiencial", Richert, 2003), planificaciones y evaluaciones realizadas a sus alumnos; (c) cuestionarios y consultas realizados ad hoc a los participantes acerca de aspectos específicos de la investigación; (d) bitácoras y apuntes de los investigadores. El material de las diferentes fuentes fue analizado a través de análisis de contenido para dar cuenta, en lo que respecta a este artículo, de una propuesta de 5 principios para el desarrollo profesional docente, los que se describirán a continuación.

\section{RESULTADOS}

\section{PRINCIPIO 1. Construir una visión común acerca del Para Qué Enseñar Ciencias. a. La Alfabetización Científica como objetivo de enseñar ciencias}

\begin{abstract}
"Ahora enseño ciencias (y quiero enseñar ciencias) para que mis estudiantes formen un pensamiento crítico-reflexivo-creativo, y puedan mejorar desde AHORA su calidad de vida, tener la aptitud de criticar y construir una solución de acuerdo a algún problema social que se relacione con las ciencias". ( $\left.\mathrm{P} 22, \mathrm{MP}^{4}\right)$. La educación en ciencias tiene como objetivo el formar individuos que debieran ser capaces de enfrentar, negociar, y tomar decisiones en situaciones cotidianas relacionadas con la Ciencia y la Tecnología.
\end{abstract}

La necesidad de contar dentro del grupo con una visión consensuada acerca del para qué enseñar ciencias fue una temática permanente. Si bien dentro del grupo ya existía una cierta idea relacionada a la alfabetización científica de la población, las discusiones y reflexiones dadas en el grupo llevaron no sólo a la convicción de que así debía ser, sino también a ciertas nociones acerca de cómo llevar este objetivo a nuestra práctica de aula. De esta manera partimos definiendo algunos de los elementos principales de la enseñanza de las ciencias: "siempre estar planteando dudas en los alumnos para que ellos vayan pensando sobre las posibles soluciones, permitir y expresarse de diversas maneras y plantear fenómenos y que los expliquen” (P15-S9), “...entregar de una forma más dinámica y entretenida el contenido a los alumnos, de manera que despierte una inquietud sobre las ciencias en su vida cotidiana, y que comiencen a cuestionarse las cosas(...)" (P15, T-O 15); elementos que permitirían desarrollar en el alumno niveles de compresión y conciencia suficientes para manejar las diversas interrelaciones entre Ciencia y Sociedad, logrando "Que descubran que no son porque sí las cosas" ( $\mathrm{P} 15, \mathrm{~T}-\mathrm{O} 1)$, generando, en contexto, aprendizajes que al alumno "(...)le permitan una mejor interacción con su entorno (en general)" (P9, T-O 1), logrando con ello que la visión del mundo por parte del alumno cambie y su entorno inmediato sea más comprensible.

\footnotetext{
MP: manuscrito en preparación.

T-O: Ticket-Out: reflexiones escritas que los docentes hacen al finalizar una reunión, respecto de alguna pregunta en particular.
} 
De esta manera vamos encontrando que nuestro quehacer implica acciones permanentes que tienen un sentido, generando una mayor comprensión de lo que entendemos por el "deber ser" de nuestra profesión: "He enfocado mi trabajo en hacer pensar a los alumnos en diferentes cosas que permiten conocer la Ciencia desde lo cotidiano. Además me he enfocado en las habilidades y no sólo en los contenidos" (P7, T-O 2). La comprensión de un nuevo sentido de enseñar ciencias implica que las expectativas en el desarrollo de nuestra enseñanza van aparejadas con una reflexión docente contextualizada a las necesidades de nuestros jóvenes, lo que involucra acciones nuevas, como la promoción del trabajo en grupo, el cual les permite a los estudiantes "entender que ellos son capaces de aprender desde su [propio] análisis un concepto científico y compararlo y debatirlo con sus compañeros para lograr respuestas" $\left(\mathrm{P} 20, \mathrm{CSC}^{6}\right)$ o la contextualización de la enseñanza mediante el uso de cuestiones socio-científicas, con el objetivo de que "los estudiantes se transformen en ciudadanos informados y puedan generar ideas y opiniones informadas" (P11, CSC), a la vez que "les permite a los estudiantes entender que muchos de los problemas sociales son causados por el hombre y sus actividades, por lo tanto, se requiere un cambio de actitud y respeto de los valores" (P17, CSC).

El entregar estas nuevas herramientas a los alumnos implica un esfuerzo que el docente debe abordar con estrategias innovadoras, metodologías contextualizadas, etc., cuestionando el cómo enseñamos conceptos, al reconocer por ejemplo: "errores conceptuales en la enseñanza del modelo atómico, enseñado habitualmente en el Plano, siendo que es una estructura que es tridimensional...” (P16, T- O 6); visualizando la necesidad de explicitar el desarrollo de habilidades científicas, por ejemplo, "relacionadas a la argumentación en que aprendan a tomar una postura-opinión respecto a algún tema socio científico que les afecte o relacione directamente" (P6, CSC), y promoviendo un impacto también en las actitudes, de modo de "hacer conciencia en el alumno y que se transmita a nivel familiar" (P8, CSC). Dar un nuevo sentido al educar en ciencias, no sólo involucra como objetivo la comprensión de conceptos científicos, desarrollo de habilidades y de actitudes en nuestros alumnos, sino también implica el lograr una cierta comprensión de lo que es la Ciencia y su relación con la Sociedad, permitiendo aportar "a la comprensión sobre nociones de la naturaleza de las ciencias (...) (Se) pueden abordar problemas de actualidad... (y) Adoptar actitudes y comportamientos sobre el impacto del desarrollo tecnológico-científico en la sociedad..." (P16, CSC). Esta relación entre la Educación en Ciencia y la transformación de la Sociedad se visualiza de manera más explícita en el siguiente punto.

b. La educación en ciencias ha de servir a la transformación y mejora de la Comunidad local

"En mi práctica me he vuelto más observadora del contexto en que viven los niños y lo que comentan, porque así creo podré visualizar las problemáticas socio-científicas locales (porque el lugar donde queda la Escuela no es mi contexto local)" (P6, T-O 3). El desarrollo de nuestras actividades docentes y el impacto que ellas pueden tener en los alumnos, permea lo que está en nuestro entorno, y nos permite impactar en la comunidad más inmediata. Esto implica no sólo contribuir a mejorar la calidad de vida de nuestros estudiantes y sus familias, sino también visualizar la posibilidad de desarrollar propuestas (para la reflexión y la prevención), y si somos más ambiciosos, aspirar a proponer soluciones a problemas concretos de la Comunidad.

CSC: encuesta acerca del uso de cuestiones socio-científicas en el aula. 
Enseñar ciencias para transformar la Sociedad, partiendo por el entorno inmediato, constituye un segundo paso en la idea de educar para la alfabetización científica de nuestros estudiantes. En una primera instancia, a los docentes participantes nos parece interesante incluir contextos sociales y culturales en nuestras clases: "Me gustó la idea de [introducir] Cuestiones Socio-científicas. Me dejó pensando en qué podría hacer respecto a lo que se puede hacer en mi Escuela" (P6, T-O 3). Esta estrategia de desarrollo profesional, basada en la reflexión colaborativa promueve el deseo de motivar a otros colegas: "Creo que no puedo dejar de motivar a mis compañeros de trabajo a realizar alguna actividad, y también a mis colegas cercanos que tienen tiempo o darles alguna idea de trabajo" (P10, T-O 2), surge también la necesidad de cuestionarse el cómo incluir a otros profesores en la contextualización social de la enseñanza de las ciencias "Cómo hacer que las historias con escenarios políticos-sociales, pueda uno validarlos y compartir con otras disciplinas para mejorar las clases en el aula(...)” (P5, T-O 2). Dado que, en general, sus colegios no promueven el trabajo en equipo, los profesores intentan por sus medios realizar trabajos interdisciplinarios: "Dentro de los 2 establecimientos donde realizo las clases no hay oportunidades formales; sin embargo entre docentes (de distintas áreas) nos embarcamos en realizar algunas cosas en conjunto; de tal manera de llevar a clases y actuar "en bloque" en cuanto a utilizar estrategias comunes" (P4, T-O 1). La enseñanza de las ciencias desde esta perspectiva entrega "La posibilidad al alumno de desarrollar un pensamiento crítico y la posibilidad de poseer argumentos científicos. Además les entrega herramientas para tomar decisiones efectivas" (P11, CSC), a la vez que permite "Contextualizar los aprendizajes de los estudiantes y valorar las ciencias en la resolución de los problemas sociales" (P17, CSC).

A partir de la visión de que la Educación en Ciencias puede ser, además de un vehículo para el desarrollo de conceptos, habilidades y actitudes, una vía para la transformación de las comunidades en donde nuestros estudiantes están insertos, es que un grupo de docentes del grupo ha desarrollado experiencias que van más allá del aula, orientadas a beneficiar a la comunidad, y que han involucrado además un trabajo interdisciplinario.

Figura 1. Algunos ejemplos de actividades interdisciplinarias con escenarios sociocientíficos y con impacto en la comunidad local

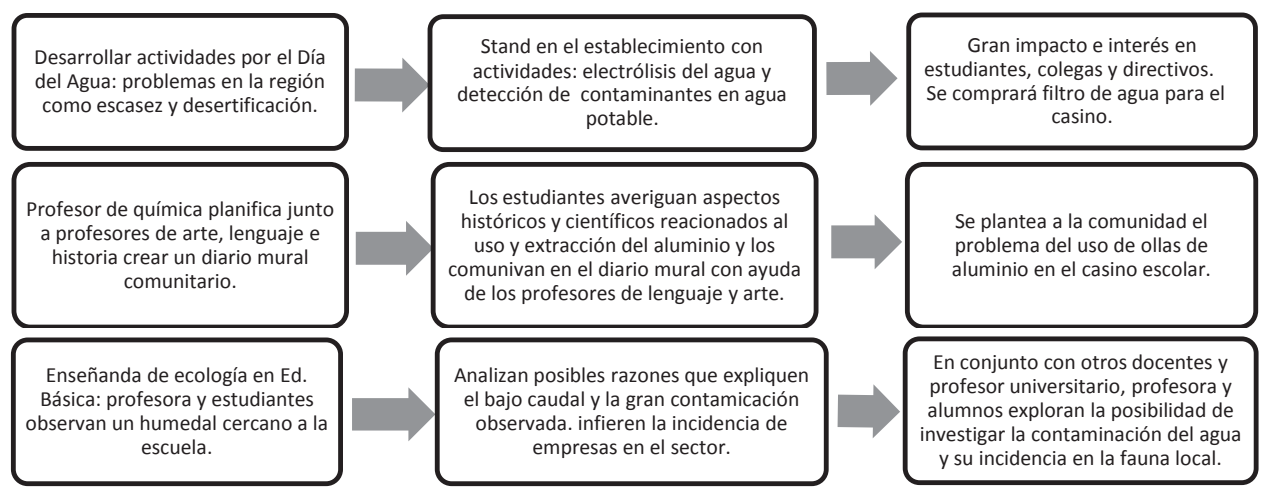


El Principio 1 corresponde evidentemente a un Principio asociado a la Educación en Ciencias en particular. Sin embargo, es posible pensar que esta misma pregunta acerca del "Para qué enseñar", podría ser el primer Principio para el desarrollo profesional en cualquier otra área disciplinar. Los siguientes principios, si bien están contextualizados a los Profesores de Ciencia, podrían ser comunes a instancias de desarrollo profesional en otras áreas.

PRINCIPIO 2. Indagar las propias prácticas a partir de las particularidades de la educación científica y tomar acciones para su mejora.

"Principalmente, lo que me cuestiono es si los alumnos comprenden la física como una rama de la ciencia que los ayuda a reflexionar y comprender el mundo que nos rodea. Y como presumo, la respuesta es negativa, me cuestiono qué estoy haciendo para transmitir ese mensaje de manera correcta" ( $\mathrm{P} 7, \mathrm{~T}-\mathrm{O}$ 1). La indagación de las propias prácticas se da a partir del cuestionamiento de aspectos concretos relacionados con la enseñanza de la ciencia en particular (biología, física, química o aspectos de la ciencia que son transversales). Lo reflexionado desde esta perspectiva es compartido en el grupo, donde se discuten posibles acciones para la mejora.

La posibilidad de un docente para mejorar sus prácticas pasa, necesariamente, por cuestionárselas (¿por qué no aprenden mis estudiantes?, ¿por qué algunos de mis estudiantes aprenden mejor o más fácilmente que otros?, etc.), e ir más allá en su indagación para encontrar respuestas que abran un rango de acción que lleven a la implementación de mejoras en los procesos de enseñanza-aprendizaje. Esta actitud indagadora de los profesores de ciencia participantes, puede sintetizarse en sus comentarios:

“(...) estoy constantemente pensando y cuestionando mi metodología de enseñanza, de manera de que la forma en que transmito el mensaje sea diferente y que realmente quede el mensaje en los estudiantes" (P7, T-O 1); "Me ha surgido la inquietud de superarme como docente, me había acostumbrado a realizar lo mismo de siempre y no me atrevía a innovar. Ahora he intentado introducir la creatividad (científica) en el aula dando desafíos a mis estudiantes y viendo cómo son capaces de hacerlo" (P11, T-O 4).

Respecto de la experiencia de indagación en nuestras prácticas, el grupo consideró que había un sinnúmero de problemas que se visualizaban en torno a la creatividad científica en la sala de clases, convirtiéndose ésta en un foco común a partir del cual cada participante abordó la indagación en la enseñanza desde su propia disciplina científica. Esto con el fin de tomar acciones concretas que produzcan una mejora de sus clases. Algunas ilustraciones de estas "indagaciones-acciones" son:

P6 (Prof. Biología, 7o básico) en su indagación encontró: "propuse construir el clásico modelo de las etapas de gestación, antes de eso tenían que comprender de qué se trataba, así es que entre videos y presentación de imágenes se intentó explicar los conceptos básicos para que pusieran en marcha su trabajo. Cuando terminaron podían nombrar las partes y funciones de placenta, cordón umbilical y amnios, pero cuando les pregunté sobre cómo se nutre, respira y elimina los desechos el feto, la reacción refleja y generalizada fue "por el cordón umbilical". Les dije: "pero el cordón es una conexión, no la fuente de nutrientes y oxígeno para el feto" y repetí la pregunta. Me di cuenta que nadie en ese momento pudo responder de manera acertada". 
Estudios Pedagógicos, vol. XL, Número Especial 1: 105-126, 2014

PRINCIPIOS DE DESARROLLO PROFESIONAL DOCENTE CONSTRUIDOS POR Y PARA PROFESORES DE CIENCIA: UNA PROPUESTA SUSTENTABLE QUE EMERGE DESDE LA INDAGACIÓN DE LAS PROPIAS PRÁCTICAS

Luego, la acción de la profesora fue “(...) por lo que les propuse [a los estudiantes] imaginar la forma en que los nutrientes y el oxígeno llegan al cordón umbilical para luego compartir sus ideas" (P6, $\left.\mathrm{HC}-2^{7}\right)$.

P9 (Prof. Física, $2^{\circ}$ medio) en su indagación encontró que sus estudiantes tenían dificultades para imaginar fenómenos de la física de su vida cotidiana, y su acción al respecto fue:

"Replico algo sencillo del acontecer diario y partir de ello realizo las preguntas. Ejemplo: Tomo una hoja de cuaderno y la suelto desde una cierta altura y pregunto por qué la hoja se mueve en la forma que lo hace. Luego tomo la misma hoja y hago con ella una pelota (esfera) y la vuelvo a soltar y vuelvo a preguntar. Vuelvo a hacer contra-preguntas a las respuesta que van dando [los estudiantes] hasta que ellos lleguen a la razón de lo acontecido (fuerza de roce)" (P9, HC).

P10 (Prof. Cs Naturales, $7^{\circ}$ básico) en su indagación encontró que "los conceptos que mis alumnos traían sobre el Universo se limitaban a describir básicamente el sistema solar, incluso considerando el Sol como la única estrella y la más grande y brillante del Cosmos". Su acción al respecto fue organizar al curso en grupos llamados "Misión Órbita de Marte" y les propuso responder "¿Cómo podemos describir las características de las principales estructuras cósmicas que forman parte del Universo y las concepciones actuales que se tienen de su origen, a los alumnos del siglo XVI?". Su acción fue pedir a sus estudiantes que trabajaran en la actividad:

"Imagina que realizan un viaje al pasado en donde los conocimientos del Universo se basaban en teorías como la teoría geocéntrica y heliocéntrica. Un viaje al pasado en donde se verán enfrentados a diferentes desafíos para explicar y describir las características de las principales estructuras cósmicas que lo forman, logrando así, que jóvenes del siglo XVI, cambien las ideas y conceptos del Universo que ellos aprenden de sus maestros". (P10, HC).

P5 (Prof. Química, $1^{\circ}$ medio) en su indagación visualizó la importancia de contextualizar su enseñanza, particularmente al tratarse conceptos abstractos como "espectros atómicos", por lo que inicia la enseñanza del tema planteando a sus estudiantes la siguiente situación:

"Los fuegos artificiales tienen su origen en China, en la región llamada Liu Yang. Allí un hombre llamado Li Tang desarrolló las primeras fórmulas, de allí se sabe es la primera mezcla explosiva llamada polvo negro, que fue procesada artesanalmente a partir de la dinastía Tang (s. VII-X) (...). La leyenda dice que un cocinero de la antigua China halló una mezcla de sulfuro, salitre y carbón de leña, mezcla de por sí, más que inflamable y posible de estallar en un espacio pequeño (...)”.

Esta situación la utiliza el docente como motivación inicial para que los estudiantes formulen hipótesis sobre el fenómeno y luego observen experimentalmente espectros de emisión a partir del calentamiento de sales (P5, HC).

Se observa en estos ejemplos que, al indagar en su práctica, cada docente encontró necesidades, problemas o desafíos a los cuales respondió con una acción específica dentro de su disciplina de enseñanza para mejorar su práctica. Estas acciones incluyeron el fin común del grupo de aplicar aspectos relacionados a la creatividad para implementar

HC: Historias de Clase. 
mejoras a su clase, pero sin dejar de lado su contexto y situación de clase. A medida que los participantes acumulaban experiencias, éstas iban compartiéndose con el grupo a través de sus reflexiones de clase. Esto llevó a establecer el principio 3.

PRINCIPIO 3. Reflexionar de manera individual y colectiva sobre el ejercicio de su práctica en un ambiente de respeto, confianza y apoyo mutuo.

"Sin duda que la reflexión que uno ejecuta es influenciada por los aciertos y fracasos relatados por otros integrantes del grupo y a partir de ello intento modificar dentro de lo posible mi práctica pedagógica" ( $\mathrm{P} 9, \mathrm{~T}-\mathrm{O}$ 3) La reflexión sobre la práctica no es un trabajo aislado, sino que ha de darse de manera colectiva. El compartir con otros en un ambiente de confianza provee de miradas diversas que enriquecen el punto de vista individual, contribuyen al cuestionamiento y apoyan el proceso de transformación de la práctica.

Un elemento clave para la transformación de las prácticas es contar con un espacio para reflexionar. Esto implica formarse y entrenarse en ello, para que no sea una acción aislada, sino que una práctica instalada. En la experiencia formativa que se presenta se puede apreciar qué entendemos por reflexión, qué la propicia y qué la hace posible.

Reflexionar sobre la práctica involucra un movimiento de las problemáticas pedagógicas desde un locus externo (problemas de los estudiantes, del establecimiento educativo, de la sociedad) a uno interno, en el que está en el docente la posibilidad de cambio. Eso se aprecia en los comentarios de la profesora: "me han llevado a reflexionar sobre qué oportunidades doy a mis alumnos para que se expresen o sean creativos" (P20, T-O 1).

La reflexión colaborativa y el escuchar a los colegas promueve también un retorno al estudiante, pues se abren los dispositivos de escucha a lo que sucede en el aula, lo que permite una mejor visión del proceso enseñanza-aprendizaje, ahora desde la óptica del alumno. Eso muestra por ejemplo la reflexión de este docente ante el comentario que realiza uno de sus estudiantes:

“dijo 'Física es muy fácil, hay que puro aprenderse las fórmulas', lo cual me hizo cuestionarme muy duramente cómo estaba llevando mi trabajo en ese momento. Me preguntaba qué era lo que realmente estaba transmitiendo, y al parecer era todo lo contrario a lo que se me había enseñado y a lo que yo pensaba que estaba transmitiendo" (P7, T-O 1).

La reflexión se instala y deja de estar reservada para ciertos momentos, individuales o colectivos, y llega a ser una práctica habitual en la que se revisan y analizan constantemente las prácticas: "ahora al pensar en alguna actividad para el curso, reflexiono acerca de lo que estoy haciendo" (P11, T-O 2).

En el discurso de los docentes participantes está presente no sólo una reflexión sobre la acción, es decir lo que se vivió o lo que se realizará, sino que también se visualiza una reflexión durante la acción, que muestra el impacto de propiciar una actitud reflexiva: "no todas las estrategias planificadas dan el resultado esperado; sin embargo hay situaciones que se generan por preguntas de los alumnos que permiten abrir espacios de enseñanzaaprendizaje más dinámicos y más pertinentes" (P4, T-O 1).

El reflexionar sobre la propia práctica conlleva un cambio a nivel identitario, particularmente se produce una reestructuración de la identidad docente, así como un reencantamiento con la docencia: "Una de las reflexiones más importantes es que me 
Estudios Pedagógicos, vol. XL, Número Especial 1: 105-126, 2014

PRINCIPIOS DE DESARROLLO PROFESIONAL DOCENTE CONSTRUIDOS POR Y PARA PROFESORES DE CIENCIA: UNA PROPUESTA SUSTENTABLE QUE EMERGE DESDE LA INDAGACIÓN DE LAS PROPIAS PRÁCTICAS

dejé llevar por la monotonía y ahora estoy intentando cambiar esto" (P11, T-O 4); "estos espacios de reflexión, me hacen sentir que lo que estoy haciendo vale la pena, cuando compruebo que mis alumnos se muestran interesados y aprendiendo" (P10, T-O 1).

Para que el reflexionar sobre la propia práctica sea efectivo, se debe contar con un espacio de confianza, un clima que posibilite el reencantarse, que permita y posibilite aprendizajes: "Es un ambiente grato, en este contexto reflexivo las ideas se entrecruzan. Siento que me gusta mucho educar, me he reencantado con mi profesión" (P10, T-O 2).

Este espacio colaborativo o colegiado resulta necesario para que los docentes adquieran una práctica reflexiva, el poder someter a juicio colectivo sus ideas, al escuchar las de sus colegas, sus reflexiones y experiencias, con aciertos y desaciertos, todo eso es en sí la reflexión más allá de lo individual, todo eso constituye la reflexión colaborativa: "El trabajo colaborativo permite trabajar en conjunto y obtener nuevas ideas y visiones para abordar las clases. Me ha permitido ver cómo abordan mis compañeros las diferentes problemáticas y qué soluciones encuentran, y analizar su resultado y sus diversas aplicaciones" (P20, T-O 4); "del trabajo colaborativo se obtienen resultados que a veces superan las capacidades individuales" (P18, T-O 4). Así, este espacio de reflexión colaborativa permite compartir experiencias positivas en el aula y los problemas propios de la educación, en donde el grupo reflexiona permitiendo que estos problemas sean superados o resignificados, en beneficio de los estudiantes:

“A mí me fortalece la relación con ustedes y el beneficio directo lo han tenido mis alumnos (...) yo veo logros en mis alumnos y para mí es constante, o sea, todos los días a cada momento y muchas veces incluso durante las mismas clases yo digo: tengo que cambiar, voy a cambiar, no, esto no sirvió, sigo con lo otro y así, yo creo que no es fácil encontrar un grupo de profesores que sean comprometidos" (P21, S16).

Sin embargo, estos espacios no están asegurados en los establecimientos educativos, y se hace menester generar dispositivos que ayuden al trabajo colaborativo: "Las oportunidades de trabajo colaborativo son casi nulas. Las instancias de reuniones entre los profesores de ciencias son solo de pasillo" (P20, T-O 1).

En resumen, los profesores participantes valoramos enormemente este espacio de formación continua porque sentimos que somos valorados y apoyados en los problemas que tenemos en nuestras aulas, sentimos que nuestra experiencia es importante, y que contribuye al aprendizaje de nuestros pares, a pesar de ser de contextos tan diferentes. El clima que se genera en este grupo permite, por una parte reencantarse con la profesión, y por otra que asistir a las reuniones se transforme en una necesidad. Por otro lado, y sin que el producto tome extrema importancia, pareciera ser que el proceso de reflexión colectiva debiera estar enlazado a una problemática común, es decir, tener metas comunes y tareas concretas que posibilitan la reflexión. Desde esta perspectiva la escritura de "historias de clase" nos ha servido como objeto de reflexión para compartir y cuestionarnos acerca de nuestro quehacer. Esto se relaciona con el siguiente principio.

PRINCIPIO 4. Valorar la autoridad de la experiencia como fuente de aprendizaje profesional.

"Por ejemplo hoy pude entender por qué exactamente no me resultaban ciertas actividades creativas. Ahora ya sé que tengo que ver bien qué pueden o no hacer los estudiantes para ver qué 
nivel de creatividad exigir" (P6, T-O 6). La experiencia, por más incipiente que sea, es una fuente de aprendizaje profesional valiosa cuando se utiliza como objeto de reflexión sobre el propio quehacer. No sólo ayuda a mejorar la propia práctica, sino también ilumina las prácticas de los demás.

En general, no hay mayor cuestionamiento cuando se plantea la teoría como fuente de autoridad y aprendizaje profesional acerca de cómo mejorar los procesos de enseñanza, sobre todo aquella teoría que emerge desde la academia a través de las investigaciones. Por su parte, valorar la experiencia como fuente de autoridad es algo un poco menos común entre los docentes y, mayoritariamente, entre los académicos, tanto si se trata de valorar la propia experiencia en docencia universitaria, como la experiencia de los docentes del sistema escolar. Considerar la propia experiencia como una fuente legítima de autoridad y de aprendizaje es relevante, y pasa por hacerse consciente del propio quehacer. La autoridad de la experiencia emana del conocimiento que reside en la acción (Munby y Russell, 1994). Esta consciencia y la valoración de la propia experiencia se potencian cuando nos abrimos a escuchar a nuestros colegas y a nuestros alumnos: "En relación a mis prácticas que me hacen reflexionar constantemente, pienso en tratar de encontrar actividades que permitan a los estudiantes mantenerlos interesados, desafiantes, entretenidos. Los eventos que gatillan principalmente esta reflexión son las constantes instancias en los que los alumnos dicen, ¿para qué me sirve esto?” (P7, T-O 1).

Un profesor consciente de su experiencia, y que la valora, puede darse el tiempo para poder comparar un estilo con otro estilo de enseñanza y para evaluar debilidades y fortalezas. Sabe analizar las experiencias de sus estudiantes, retoma los factores sociales, ambientales y culturales que rodean al mismo, y logra visualizar el camino a seguir hacia un buen proceso de aprendizaje, construyendo además una autoestima que apoya el realizar cambios reales en su labor docente: "Se produce una ampliación del campo de reflexión y se producen inquietudes que se visualizan con soluciones o respuestas a distintas etapas de la reflexión" (P19, T-O 3).

Un profesor consciente de su experiencia y del valor de ésta está observando lo que sucede en su aula: “¿Cómo desarrollar la capacidad de razonamiento abstracto? Me he dado cuenta que a pesar de modificar mis prácticas, no he logrado una mejora notoria en este punto en mis alumnos" (P14, T-O 1). Escucha a sus estudiantes y reconoce oportunidades de enseñanza: "Yo creo que el diálogo de la sala de clase es un diálogo tan rico (...) es vivo, es activo, es un diálogo que si tú no estás despierto, despierto en todos los sentidos, se te pasó y lo desaprovechaste y perdiste la oportunidad" (P4, S16). Y reconoce en la experiencia una fuente de futuros aprendizajes: "Me he dado cuenta que me he visto en contra del tiempo y termino realizando lo más sencillo que es pasar materia. Esto lo afronto con paciencia porque sé que la experiencia me irá dando más y mejores herramientas" (P7, T-O 2).

Un aspecto importante que contribuye a utilizar la experiencia como fuente de aprendizaje profesional, y que sobretodo permite compartirla, es escribir sobre ella. En el grupo participante de este estudio esto se ha logrado gracias a la escritura de nuestras historias de clase $^{8}$, donde en no más de dos páginas se resume una experiencia de aula, señalando brevemente el contexto, el objetivo de la clase, las actividades realizadas, la

El proyecto australiano PEEL, anteriormente mencionado, trabaja a partir de estos testimonios y reflexiones y tiene una muy amplia base de datos (ver www.peelweb.org). 
Estudios Pedagógicos, vol. XL, Número Especial 1: 105-126, 2014

PRINCIPIOS DE DESARROLLO PROFESIONAL DOCENTE CONSTRUIDOS POR Y PARA PROFESORES DE CIENCIA: UNA PROPUESTA SUSTENTABLE QUE EMERGE DESDE LA INDAGACIÓN DE LAS PROPIAS PRÁCTICAS

respuesta de los estudiantes y una reflexión final acerca de todo el proceso. Idealmente, incorporamos algún tipo de evidencia de lo que sucede con los estudiantes (fotos de los cuadernos, frases u opiniones que dicen o escriben los estudiantes, etc.). Una vez escritas, las historias de clase se comparten en pequeños grupos y, dependiendo del objetivo, se analizan a través de ciertas preguntas como ¿Qué tienen en común estas actividades? ¿Cómo podemos mejorar las evidencias que recogimos en estas historias?, etc. De esta manera, la experiencia propia no sólo se vuelve fuente de autoridad y de aprendizaje profesional para el docente que la vive, sino también para aquél que la escucha y analiza, aunque no la haya vivido directamente.

PRINCIPIO 5. Promover y valorar la participación de profesores provenientes de una diversidad de contextos y niveles en los que se ejerce la docencia.

"Beneficios [del trabajo colaborativo]: Cuestionamiento total de mis prácticas. La comprensión y cada vez mayor certeza de lo enriquecedor que es el compartir con otros, sobretodo de contextos diferentes" ( $\mathrm{P} 1, \mathrm{~T}-\mathrm{O} 2)$. La diversidad de contextos y niveles en los que trabajan los docentes que comparten sus experiencias es fundamental para lograr una mayor comprensión de los procesos de aprendizaje y enseñanza, en todos los niveles. Esta diversidad debiera incluir como participantes activos, además de docentes del sistema escolar, a docentes en formación y a docentes universitarios, especialmente a los formadores de profesores.

Todos los profesores tenemos ideas sobre cómo obtener buenos resultados con nuestros estudiantes, y no sólo eso, de acuerdo con nuestras propias experiencias, también tenemos representaciones acerca de cómo debiéramos mejorar nuestras propias prácticas. Sin embargo, no es menos cierto que la mayoría de las veces también pensamos que son nuestros contextos, o el currículum, o la falta de recursos u otros obstáculos, las principales causas de que no podamos hacer innovaciones. Entre algunas otras, particularmente en ciencias, una de las causas principales de estas creencias de los profesores está radicada en el hecho de que nuestro trabajo se transforma en una isla al interior de nuestras instituciones: "En general, todos los profesores de nuestro liceo hacemos ver esta problemática [la falta de trabajo colaborativo], que incide directamente en los aprendizajes de los alumnos; no hay una integración de los contenidos, perdiendo así mayor significancia" (P20, T-O 1).

Desde esta perspectiva, el espacio de reflexión grupal se caracteriza principalmente por permitir la interacción de voces de profesores que provienen desde distintos contextos y niveles. Uno de sus efectos es que ha permitido una nueva valoración personal del trabajo, y la visualización de nuevos desafíos que se incorporan al campo de experiencias de los docentes: "Siento que puedo afrontarlos mejor [los desafíos], ya que en el grupo se escuchan distintas experiencias y siento que he ido aprendiendo de ellas haciendo. Esto ha hecho que quiera siempre desafiarme y mejorar mi desempeño laboral" (P11, T-O 2). La valoración de la diversidad a partir del conocimiento de experiencias provenientes de diferentes contextos, disciplinas y niveles lleva a una visión ampliada del problema de aprender ciencias:

"Me ha servido para analizar mis prácticas y cómo abordaba las clases desde una postura donde consideraba mi asignatura como una isla, hasta darme cuenta que todos los procesos de aprendizaje de los alumnos están entrelazados y debemos considerar una visión compartida de cómo desarrollar el trabajo en clase, no solo considerando los conceptos previos de nuestros 
estudiantes con respecto a nuestros contenidos, sino también sus competencias matemáticas, su comprensión lectora y sus diferentes habilidades". (P20, T-O 1).

Desde el punto de vista de los docentes universitarios que participan en la formación de profesores, discutir junto a los docentes del sistema escolar acerca de sus propias prácticas universitarias y a la vez escuchar las experiencias de profesores tanto de nivel secundario como primario, ha ayudado a cuestionar su propia docencia en el contexto de la formación inicial y continua: "Si, han surgido nuevos cuestionamientos, sobre todo relacionados con cómo, en el contexto de la formación inicial de profesores, facilitar el que los estudiantes acorten la brecha entre teoría y la práctica (...). Creo que en todo esto la conversación con los otros colegas es fundamental" (P1, T-O 2). Por otra parte, para aquellos docentes universitarios que no trabajan en el contexto escolar, conocer la experiencia de los docentes, tanto de educación secundaria como primaria, ha permitido resignificar, visualizar y traducir constructos teóricos a experiencias concretas, las que luego son utilizadas como ejemplo por los mismos docentes en otros procesos de formación docente, inicial y continua:

"A raíz de la participación en un Congreso (ESERA 2013) yo había conocido un poco más acerca de los Socio-scientific-Issues [Cuestiones Sociocientíficos], tema que compartí en el grupo. A propósito de ello se generó una interesante discusión acerca de cuál es finalmente el significado de enseñar Ciencias, y cómo hacemos que la Educación en Ciencias contribuya tanto a la comprensión del mundo natural, como a la mejora de la calidad de vida de nuestros estudiantes y sus comunidades. Uno de los primeros relatos que me impresionó en este sentido fue el de una de las profesoras de primaria. Ella contó que sus estudiantes de $6^{\circ}$ básico habían estado investigando las características de un río que corre cerca de la Escuela y del lugar que habitan los niños (...). La experiencia de esta profesora, y toda la discusión que generó su ejemplo, produjo un primer cambio en mi visión de Alfabetización Científica (...). Lo anterior me llevó a reflexionar, discutir con otros colegas de la Universidad y leer más bibliografía acerca de las maneras de contextualizar la enseñanza de las ciencias” (P1, MP).

Del mismo modo, la experiencia que los docentes universitarios han podido llevar al grupo desde la Academia, ha contribuido a la innovación de las prácticas en la Escuela:

"Otro de los temas que surgió en el grupo de investigación y que me ha ayudado a cambiar ha sido el de los temas socio-científicos (...) trabajar con los estudiantes problemáticas sociocientíficas abre el espectro y ataca dos flancos que son la reflexión de la física aplicada en cosas cotidianas y relevantes no solo para los estudiantes sino para la sociedad, y además se trabaja en ellos de manera de que puedan desarrollar la creatividad, ya que se presentan como problemas en donde ellos deben proponer y generar ideas (P7, MP).

Finalmente, también en los profesores en formación, estudiantes de últimos años de Pedagogía en Ciencias, se han gatillado procesos de reflexión, que los llevan a estar mejor preparados para enseñar:

"Si no hubiese visto la evidencia viva de que hay profesores que realizan sus clases de modo indagatorio, reflexionan antes-durante-después de cada clase, valoran su profesión, que aprenden cada día cómo mejorar los aprendizajes de sus estudiantes, contextualizan los contenidos a la vida de ellos, les interesan y buscan estrategias para conocerlos, jamás hubiese creído que sea posible lograr que los estudiantes construyan su conocimiento” (P22, MP). 
Lo mismo en el caso de los profesores novatos: "Las reuniones de trabajo y reflexión han permitido obtener las herramientas que en un momento faltan, sobre todo a profesores sin experiencia en la docencia" ( $P 7, M P)$.

Por último, al reconocer la riqueza de la diversidad de los contextos de trabajo del grupo, se observa que los docentes se han permitido otras posibilidades para el desarrollo de trabajo colaborativo, a través de redes que se generan entre los participantes del grupo. Así, una de las docentes de segundo ciclo básico relata:

"En un proyecto colaborativo con la asignatura de química del establecimiento nace la idea de sumar fuerzas en esta problemática y se da inicio a un Proyecto Científico Escolar de Explora con alumnos de $2^{\circ}$ Medio, contando con la asesoría de un profesor del Instituto de Química de la $P U C V^{9}$. El proyecto consiste en analizar muestras contaminantes de metales pesados del río Aconcagua en varios puntos a lo largo del curso por donde pasa el río hasta la desembocadura y estudiar posibles efectos en el ecosistema del humedal que se encuentra alli”" (P10, MP).

En este mismo ámbito, un profesor de ciencias del Sistema Escolar y un docente de la Universidad tuvieron la oportunidad de desarrollar, durante el segundo semestre de 2013, un curso integrado de Física y Química llamado "Juguetes, Ciencias y TICs" en un programa de talentos académicos, dirigido a estudiantes de segundo ciclo básico. Esta experiencia, además de mantenerse en el tiempo, dio origen a otros dos cursos en el mismo programa ("Química: la magia de la ciencia cotidiana" y "Problemáticas sociocientíficas: su impacto en el entorno natural"), donde uno de los docentes de química secundarios trabaja en conjunto con uno de los estudiantes de Pedagogía en Biología, ambos integrantes del grupo.

\section{DISCUSIÓN}

Los 5 principios propuestos abarcan el ámbito personal, social y profesional de profesores de ciencia, y sugieren la necesidad de abordar de manera sinérgica aspectos que son clave para el desarrollo profesional docente. Así, al construir una visión común acerca del para qué enseñar ciencias (Principio 1), logramos visualizar un propósito mayor y sus desafíos. Al indagar y tomar acciones de mejora respecto a nuestra propia práctica con esta visión en mente, acumulamos y analizamos experiencias desde nuestra disciplina particular de enseñanza (Principio 2), las cuales usamos para reflexionar individualmente y compartir reflexionando colaborativamente (Principio 3). De allí que, valorando la autoridad de nuestra experiencia (Principio 4), nos desarrollamos profesionalmente identificando nuevas inquietudes y compartiéndolas respecto a la propia práctica, donde la diversidad de contextos y niveles de enseñanza son un valor agregado de conocimiento y experiencia (Principio 5). Esta interacción continua entre lo personal de nuestra práctica y lo colectivo es lo que nos mantiene activamente involucrados en un desarrollo profesional que, para varios de los integrantes, se ha vuelto una travesía que ya lleva 4 años, mientras para otros que recién se integran, es el comienzo de un interesante camino de aprendizaje profesional.

Los principios aquí planteados también son apoyados por investigaciones anteriores. Varios estudios indican que la formación continua efectuada de manera colaborativa,

Dicho profesor participó inicialmente del grupo. 
reflexiva e indagatoria respecto de la propia experiencia de aula, es más efectiva que la tradicional en la adopción de nuevas prácticas. Camburn (2010) con una muestra de 80 colegios en EE.UU., encontró que la implementación de un programa integral de reforma en ellos fue más efectiva de manera alternativa (aprendizaje colaborativo basado en sus propias prácticas) que tradicional (aprendizaje no colaborativo y aislado de las propias prácticas), resultando una relación estadísticamente fuerte y positiva respecto a la reflexión docente (lo que respalda el Principio 3). Goodnough (2010), en un estudio de caso de una profesora de ciencias en un contexto de investigación-acción colaborativa, da luces del rol de la indagación colaborativa y las posibilidades de desarrollo del conocimiento docente. La autora encontró que mientras la profesora usaba conocimiento de otros, también generaba conocimiento construyendo y reconstruyendo el conocimiento de su experiencia de aula (Principio 4); además examinó sus creencias sobre la enseñanza-aprendizaje de las ciencias, permitiéndole conocer facetas de su propio conocimiento profesional (Principio 2). Lebak y Tinsley (2010) por otra parte, reportan la transformación de 3 profesores de ciencia que deseaban volverse más efectivos en involucrar a sus estudiantes en el aprendizaje de las ciencias (Principio 1). En el marco de un modelo de investigación-acción con un enfoque en la reflexión colaborativa (Principio 3), la transformación resultante de estos profesores fue desde una enseñanza de las ciencias basada en los textos de estudio de los estudiantes, a una centrada en los estudiantes con un enfoque indagatorio.

En relación a lo planteado al comienzo de este artículo, el camino recorrido por este conjunto de docentes se presenta también de manera muy similar a lo anteriormente descrito respecto de cómo se van desarrollando, de manera paulatina, los ámbitos personal, social y profesional de los docentes según la investigación de Bell y Gilbert (Simon y Campbell, 2012). Del mismo modo, se pueden identificar en el proceso tanto las 8 condiciones de Hoban descritas al inicio de este artículo, bajo las cuales se promueve el aprendizaje profesional docente, como varios de los principios para un desarrollo profesional efectivo señalados por Loucks-Horsley, Styles y Hewson (1996), particularmente los principios 2, 3,4 y 5 que plantean estos autores.

No obstante, es interesante destacar que los principios que aquí se presentan, por las características particulares de la composición del grupo, así como por el hecho de emerger desde un contexto local, aportan con elementos que están ausentes o menos explícitos en los principios y condiciones planteados por los autores señalados, que hacen de nuestra propuesta algo pertinente a nuestra realidad local. Por ejemplo, respecto de considerar más explícitamente la especificidad de la disciplina a enseñar (Ciencias, y no otra disciplina): es posible identificar en los principios 1 y 2 planteados en este trabajo referencias a ello. Si bien el primer principio de Loucks-Horsley, Styles y Hewson (1996) considera la necesidad de partir con una imagen de enseñanza efectiva de las ciencias, ésta se refiere a cómo enseñar ciencias, pero no al para qué enseñar ciencias (Principio 1 de este trabajo). En nuestro caso, estar situados en un contexto latinoamericano de país en vías de desarrollo, nos lleva a la necesidad de ser mucho más explícitos y enfáticos respecto de para qué estamos enseñando ciencias y la manera en que esperamos que la educación en ciencias contribuya a la resolución de problemas que son propios de nuestra realidad, como los presentes en los ámbitos medioambiental, energético, salud, etc. En este sentido, este principio debiera ser un elemento basal de las políticas de formación continua en el área científica, si es que realmente queremos alfabetizar científicamente a nuestra población, de modo de que seamos capaces de asumir los desafíos científicos y tecnológicos que vive nuestra sociedad y elevar nuestra calidad de vida como país. 
Estudios Pedagógicos, vol. XL, Número Especial 1: 105-126, 2014

PRINCIPIOS DE DESARROLLO PROFESIONAL DOCENTE CONSTRUIDOS POR Y PARA PROFESORES DE CIENCIA: UNA PROPUESTA SUSTENTABLE QUE EMERGE DESDE LA INDAGACIÓN DE LAS PROPIAS PRÁCTICAS

Por otra parte, relevar la autoridad de la experiencia (Principio 4) de forma explícitaa través de la escritura y análisis de historias de clase constituye un piso a partir del cual una comunidad comienza a generar conocimiento profesional, que es factible de compartir y utilizar para los procesos de desarrollo profesional en otros contextos, incluyendo la formación inicial. Por último, la diversidad de contextos y niveles de formación (Principio 5) debiera contribuir directamente a superar los problemas que planteaban Núñez, Arévalo y Ávalos (2012) respecto del desencuentro entre los ámbitos de formación inicial y continua, y los ámbitos del saber (teoría y práctica) y las unidades formadoras (Universidad y Escuela). En el caso de los formadores de profesores, el hecho de ser nosotros mismos objetos y sujetos de investigación, y compartir en un mismo nivel nuestras prácticas con las de los colegas de establecimientos escolares y en formación, nos permitió tener una mirada más amplia de lo que supone el proceso de formación docente, incluyendo nuestro propio proceso de aprender a enseñar, contribuyendo a una visión acerca de cómo debiera ser la relación entre la Universidad y la Escuela.

Finalmente, cabe señalar que si bien los principios que aquí se plantean son dinámicos y están en permanente discusión, pensamos que pueden ser útiles para orientar los procesos de desarrollo profesional, al menos en el contexto latinoamericano. Una formación continua de profesores de ciencia que incorpore los 5 principios propuestos no sólo puede aportar a la implementación de reformas un mayor conocimiento de la propia enseñanza y mejoras de la enseñanza y aprendizaje de las ciencias de forma efectiva a todos los niveles, sino que promete un cambio más profundo y autosustentable: el de la transformación de los docentes y sus estudiantes.

\section{REFERENCIAS BIBLIOGRÁFICAS}

Albertini, R., Cárdenas-Jirón, G., Babel, J., Díaz Véliz, G., Eyzaguirre, J., Labra, A. y Lewin, R. (2005). Enseñanza de las ciencias a nivel escolar y formación en ciencia en el pregrado universitario. En T. Ureta, J. Babul, S. Martínez y J. Allende (Eds.) Análisis y Proyecciones de la Ciencia Chilena 2005. Academia Chilena de Ciencias. Recuperado desde http://www.academia-ciencias.cl/wp/wpcontent/uploads/2010/08/001_Presentacion.pdf

Ávalos, B. (2002). La formación docente continua en Chile. Desarrollo, logros y limitaciones. En Formación docente: un aporte a la discusión. La experiencia de algunos países (pp. 35-57). Chile: UNESCO/OREALC.

Ávalos, B. (2011). Teacher professional development in Teaching and Teacher Education over ten years. Teaching and Teacher Education, vol.27, n.1, 10-20.

Bell, B. (1998). Teacher Development in Science Education. In T. Fraser \& K. Tobin (Eds.), International Handbook of Science Education (pp. 681-693). Kluwer: Academic Publishers.

Camburn, E.M. (2010). Embedded teacher learning opportunities as a site for reflective practice: an exploratory study. American Journal of Education, vol.116, n.4, 463-489.

Chou, C. (2011). Teachers professional development: investigating teacher's learning to do action research in a professional learning community. The Asia-Pacific Education Researcher, vol.20, n.3, 412-437.

Chval, K., Abell, S., Pareja, E., Musiku, K., \& Ritzka, G. (2008). Science and Mathematics Teachers' Experiences, Needs, and Expectations Regarding Professional Development. Eurasia Journal of Mathematics, Science and Technology Education, vol.4, n.1, 31-43.

Clough, M., Berg, C., \& Olson, J. (2009). Promoting effective science teacher education and science teaching: a framework for teacher decision-making. International Journal of Science and 
Estudios Pedagógicos, vol. XL, Número Especial 1: 105-126, 2014 PRINCIPIOS DE DESARROLLO PROFESIONAL DOCENTE CONSTRUIDOS POR Y PARA PROFESORES DE CIENCIA: UNA PROPUESTA SUSTENTABLE QUE EMERGE DESDE LA INDAGACIÓN DE LAS PROPIAS PRÁCTICAS

Mathematics Education, vol.7, n.4, 821-847.

Cochran-Smith, M. y Lytle, S. (2003). Más allá de la certidumbre: adoptar una actitud indagadora sobre la práctica. En A. Lieberman y L. Millar (Eds.), La indagación como base de la formación del profesorado y la mejora de la educación (pp. 65-80). Barcelona: Ediciones Octaedro.

Cofré, H., Camacho, J., Galaz, A., Jiménez, J., Santibáñez, D. y Vergara, C. (2010). La educación científica en Chile: debilidades de la enseñanza y futuros desafíos de la educación de profesores de ciencia. Estudios Pedagógicos, vol.35, n.2, 279-293.

De Lella, C. (1999). Modelos y tendencias de la Formación Docente. Trabajo presentado en el I Seminario Taller sobre Perfil del Docente y Estrategias de Formación, OEI. Recuperado desde http:// www.oei.es/cayetano.htm

Echeverría, P. (2010). El papel de la docencia universitaria en la formación inicial de profesores. Calidad en la Educación, n.32, 150-165.

González-Weil, C., Martínez, M., Martínez, C., Cuevas, K. y Muñoz, L. (2009). La educación científica como apoyo a la movilidad social: Desafíos en torno al rol del profesor secundario en la implementación de la indagación científica como enfoque pedagógico". Estudios Pedagógicos, vol.35, n.1, 63-78.

González-Weil, C., Cortéz, M., Bravo, P., Ibaceta, Y., Cuevas, K., Quiñones, P., Maturana, J. y Abarca, A. (2012). La indagación científica como enfoque pedagógico: estudio sobre las prácticas innovadoras de docentes de ciencia en EM (Región de Valparaíso). Estudios Pedagógicos, vol.38, n.2, 85-102.

González-Weil, C., Cortéz, M., Pérez, J., Bravo, P. y Ibaceta, Y. (2013). Construyendo dominios de encuentro para problematizar acerca de las prácticas pedagógicas de profesores secundarios de Ciencias: Incorporando el modelo de Investigación-Acción como plan de formación continua. Estudios Pedagógicos, vol.39, n.2, 129-146.

Goodnough, K. (2010). Teacher learning and collaborative action research:generating a "knowledge-of-practice" in the context of science education. Journal of Science Teacher Education, vol.21, n.8, 917-935.

Latorre, M. (2005). Continuidades y rupturas entre Formación Inicial y ejercicio profesional docente. Revista Iberoamericana de Educación, n.36, 2, 1-11.

Lebak, K., \& Tinsley, R. (2010). Can inquiry and reflection be contagious? Science teachers, students, and action research. Journal of Science Teacher Education, vol.21, n.8, 953-970.

Loucks-Horsley, S., Styles, K., \& Hewson, P. (1996). Principles of effective professional development for mathematics and science education: A synthesis of standards. National Institute for Science Education, vol.1, n.1, 1-6.

Loughran, J. (2007). Science Teacher as Learner. In S. Abell \& N. Lederman (Eds.). Handbook of Research on Science Education (pp. 1043-1065). Oxford, UK: Taylor \& Francis.

Mellado, V. (2001). ¿Por qué a los profesores de ciencias nos cuesta tanto cambiar nuestras concepciones y modelos didácticos? Revista Interuniversitaria de Formación del Profesorado, n.40, 17-30.

Munby, H., \& Russell, T. (1994). The authority of experience in learning to teach: Messages from a physics methods class. Journal of Teacher Education, vol.45, n.2, 86-95.

Munby, H., \& Russell, T. (2003). Epistemology and Context in Research on Learning to Teach Science. In B. Fraser \& K. Tobin, (Eds.), International Handbook of Science Education (pp. 643665). Dordrecht: Kluwer Academic Publishers.

Muñoz, G. y Vanni, X. (2008). Rol del estado y de los agentes externos en el mejoramiento de las escuelas: análisis en torno a la experiencia chilena. Revista Iberoamericana sobre Calidad, Eficacia y Cambio en Educación, vol.6, n.4, 47-68.

Núñez, M., Arévalo, A. y Ávalos, B. (2012). Profesionalización docente: ¿Es posible un camino de convergencia para expertos y novatos? Revista Electrónica de Investigación Educativa, vol.14, n.2, 10-24. 
Estudios Pedagógicos, vol. XL, Número Especial 1: 105-126, 2014

PRINCIPIOS DE DESARROLLO PROFESIONAL DOCENTE CONSTRUIDOS POR Y PARA PROFESORES DE CIENCIA: UNA PROPUESTA SUSTENTABLE QUE EMERGE DESDE LA INDAGACIÓN DE LAS PROPIAS PRÁCTICAS

Ponte, P. (2010). Action Research as a Tool for Teachers' Professional Development. In P. Peterson, E. Baker, \& B. McGaw (Eds.), International Encyclopedia of Education (3 ${ }^{\text {rd }}$ ed.) (pp. 540547). Oxford: Elsevier.

Richert, A. (2003). La narrativa como texto experiencial: incluirse en el texto. En A. Lieberman y L. Miller, La indagación (pp. 193-208). Barcelona: Octaedro.

Sadler, T. (2011). Situating Socio-scientific Issues in classrooms as a means of achieving goals of Science Education. In T. Sadler (Ed.), Socio-scientific Issues in the Classroom: teaching, learning, and research (pp. 1-10). The Netherlands: Springer Press.

Simon, S., \& Campbell, S. (2012). Teacher Learning and Professional Development in Science Education. In B. Fraser, K. Tobin, \& C. McRobbie (Eds.), Second International Handbook of Science Education (Vol. 24). Springer International Handbooks of Education. doi: 10.1007/978-1-40209041-7

Vescio, V., Ross, D., \& Adams, A. (2008). A review of research on the impact of professional learning communities on teaching practice and student learning. Teaching and Teacher Education, vol.24, n.1, 80-91. 